01

\title{
Расчёт сверхтонкой магнитной аномалии в многоэлектронных атомах
}

\author{
(C) Е.А. Коновалова ${ }^{1}$, Ю.А. Демидов ${ }^{1,2}$, М.Г. Козлов ${ }^{1,2}$ \\ ${ }^{1}$ Петербургский институт ядерной физики им. Б.П. Константинова Национального исследовательского центра \\ „Курчатовский институт“, \\ 188300 Гатчина, Россия \\ ${ }^{2}$ Санкт-Петербургский государственный электротехнический университет „ЛЭТИ“, \\ 197376 Санкт-Петербург, Россия \\ e-mail: lenaakonovalova@gmail.com
}

Поступила в редакцию 06.02.2020 г.

В окончательной редакции 30.03.2020 г.

Принята к публикации 04.05.2020 г.

Прецизионные измерения отношения констант сверхтонкой структуры для состояний $s_{1 / 2}$ и $p_{1 / 2}$ позволяют оценить разность сверхтонких магнитных аномалий для этих уровней. Настоящая работа посвящена восстановлению абсолютных величин сверхтонких магнитных аномалий из их разности. Учет поправки на сверхтонкую магнитную аномалию к $g$-фактору позволяет более чем на порядок увеличить точность определения $g$-факторов короткоживущих изотопов.

Ключевые слова: сверхтонкая магнитная аномалия, эффект Бора-Вайскопфа, эффект Брейта-Розенталь.

DOI: $10.21883 /$ OS.2020.10.50009.36-20

\section{1. Введение}

В последние годы точность, достигаемая в экспериментах по лазерной спектроскопии, в совокупности с развитием атомной теории позволила перейти к проверке различных ядерных моделей $[1,2]$. Магнитные дипольные константы сверхтонкой структуры (СТC) $A$ зависят от распределения заряда и намагниченности внутри атомного ядра. В приближении точечного ядра отношение постоянных СТС для двух изотопов равно отношению их $g$-факторов $g_{I}=\frac{\mu}{\mu_{N}}$, где $\mu$ и $I-$ магнитный момент и спин ядра; $\mu_{N}$ - ядерный магнетон. Рассматривая ядра конечных размеров, необходимо учитывать распределение намагниченности по ядру и зависимость электронной волновой функции от радиуса ядра. Первая поправка называется магнитной (Бора-Вайскопфа [3]), вторая - зарядовой (Брейта-Розенталь [4,5]). Эти поправки нарушают пропорциональность между константами СТС и ядерными $g$-факторами, что получило название сверхтонкой магнитной аномалии (СМА):

$$
{ }^{1} \Delta^{2} \equiv \frac{A^{(1)} g_{I}^{(2)}}{A^{(2)} g_{I}^{(1)}}-1,
$$

где $A^{(1)}, A^{(2)}$ - константы СТС двух изотопов, $g_{I}^{(1)}$ и $g_{I}^{(2)}$ - соответствующие $g$-факторы.

При извлечении ядерных $g$-факторов из экспериментальных данных поправкой на СМА часто пренебрегают из-за отсутствия теоретических оценок на её величину. С другой стороны, поправка на СМА обычно мала (менее 1\%) [6], и только недавно измерения для короткоживущих ядер достигли такого уровня точности. Также исследования констант СТС могут послужить полезным инструментом для понимания явления сосуществования форм в атомных ядрах [7]. Таким образом, разработка новых методов атомных вычислений констант СТС, учитывающих СМА, становится актуальной и своевременной.

\section{2. Поправки на конечный размер ядра}

Наблюдаемые константы сверхтонкой структуры $A$ могут быть записаны в следующей форме [8]:

$$
A=g_{I} \mathscr{A}_{0}(1-\delta)(1-\epsilon) .
$$

Здесь $g_{I}-$ ядерный $g$-фактор, $g_{I} \mathscr{A}_{0}-$ константа сверхтонкой структуры в случае точечного ядра, $\delta$ и $\epsilon-$ безразмерные поправки на распределение заряда (BR) и намагниченности $(\mathrm{BW})$ в ядре. Константа $\mathscr{A}_{0}$ не зависит от ядерного $g$-фактора.

В случае водородоподобных ионов выражение для $\mathcal{A}_{0}$ известно в аналитической форме $[9,10]$ :

$$
\mathcal{A}_{0}=\frac{\alpha(\alpha Z)^{3}}{j(j+1)} \frac{m}{m_{p}} \frac{\varkappa\left(2 \varkappa\left(n_{r}+\gamma\right)-N\right)}{N^{4} \gamma\left(4 \gamma^{2}-1\right)} m c^{2} \text {. }
$$

Здесь $\alpha$ - постоянная тонкой структуры, $Z-$ заряд ядра, $m$ и $m_{p}$ - массы электрона и протона, $j$ полный момент электрона, $\varkappa=(l-j)(2 j+1)-$ релятивистское квантовое число, $N=\sqrt{\left(n_{r}+\gamma\right)^{2}+(\alpha Z)^{2}}-$ эффективное главное квантовое число, $n_{r}=n-|\varkappa|-$ радиальное квантовое число, $n-$ нерелятивистское главное квантовое число, $\gamma=\sqrt{\varkappa^{2}-(\alpha Z)^{2}}$. Заметим, что $\mathscr{A}_{0}$ для состояний с $j=\frac{1}{2}$ обращается в бесконечность при $\gamma=\frac{1}{2}$ или $Z \approx 118$. 
При одинаковых $n$ отношения $\mathscr{A}_{0, s_{1 / 2}} / \mathscr{A}_{0, p_{1 / 2}}$ и $\mathscr{A}_{0, p_{1 / 2}} / \mathscr{A}_{0, p_{3 / 2}}$ равны:

$$
\begin{gathered}
\frac{\mathscr{A}_{0, s_{1 / 2}}}{\mathscr{A}_{0, p_{1 / 2}}}=3+2\left(\frac{\alpha Z}{n-1+\gamma_{1 / 2}}\right)^{2} ; \\
\frac{\mathscr{A}_{0, p_{1 / 2}}}{\mathscr{A}_{0, p_{3 / 2}}}=\frac{\gamma_{3 / 2}\left(4 \gamma_{3 / 2}^{2}-1\right)}{2 \gamma_{1 / 2}\left(4 \gamma_{1 / 2}^{2}-1\right)}\left(1+3(\alpha Z)^{2} \frac{5 n-4}{20 n^{2}}\right) .
\end{gathered}
$$

Здесь учтено, что $\gamma$ для состояний $s_{1 / 2}$ и $p_{1 / 2}$ совпадают, а для состояний $p_{1 / 2}$ и $p_{3 / 2}$ отличаются друг от друга. Уравнение (4) согласуется с результатами, полученными для многоэлектронных систем в квазиклассическом приближении [11].

Будем рассматривать ядро как равномерно заряженный и намагниченный шар радиуса $R=\left(\frac{5}{3}\left\langle r^{2}\right\rangle\right)^{1 / 2}$, где $r_{\text {rms }}=\left\langle r^{2}\right\rangle^{1 / 2}-$ среднеквадратичный радиус ядра. Распределение намагниченности по ядру обусловлено спиновой поляризацией нуклонов и орбитальным движением протонов.

Плотность заряда внутри ядра относительно стабильна для различных изотопов [12], тогда как распределение намагниченности сильно зависит от спина ядра и нуклонной конфигурации каждого изотопа. Для параметризации этих эффектов согласно работам $[13,14]$ использовался ядерный множитель $d_{\text {nuc }}$. Тогда поправки $\delta$ и $\epsilon$ для выбранного $Z$ и электронного состояния могут быть записаны как [15]

$$
\begin{gathered}
\delta(R)=b_{N}\left(R / \lambda_{C}\right)^{2 \gamma-1}, \\
\epsilon\left(R, d_{\text {nuc }}\right)=b_{M} d_{\text {nuc }}\left(R / \lambda_{C}\right)^{2 \gamma-1} .
\end{gathered}
$$

Здесь $b_{N}$ и $b_{M}$ - безразмерные параметры, не зависящие от радиуса и структуры ядра, которые могут быть найдены численно; $\lambda_{C}-$ комптоновская длина волны электрона $\left(\lambda_{C}=\frac{\hbar}{m_{\rho} c}\right)$. Ядерный множитель определен так, чтобы $d_{\text {nuc }}=0$ соответствовал точечному магнитному диполю в центре ядра, $d_{\text {nuc }}=1-$ равномерно намагниченному шару радиуса $R$.

Параметризация констант СТС уравнениями (2), (5) включает три ядерные $\left(g_{I}, d_{\text {nuc }}, R\right)$ и три атомные $\left(\mathscr{A}_{0}\right.$, $\left.b_{N}, b_{M}\right)$ характеристики. Атомные параметры одинаковы для различных изотопов. Информацию о ядре можно извлечь из экспериментально измеренных констант СТС, если рассчитаны атомные параметры. В изотопическом ряду радиус ядра $R$ обычно плавно меняется и лишь на несколько процентов, таким образом поправка $\delta$ практически одинакова для всех изотопов выбранного атома.

Для расчета констант СТС необходимо задать ядерные параметры:

$$
\begin{gathered}
A\left(g_{I}, d_{\mathrm{nuc}}, R\right)=g_{I} \mathscr{A}_{0}\left(1-b_{N}\left(R / \lambda_{C}\right)^{2 \gamma-1}\right) \\
\times\left(1-d_{\mathrm{nuc}} b_{M}\left(R / \lambda_{C}\right)^{2 \gamma-1}\right) .
\end{gathered}
$$

Для того, чтобы найти атомные параметры $\mathcal{A}_{0}, b_{N}$ и $b_{M}$ из (2), (5), достаточно варьировать значения $d_{\text {nuc }}$ и
$R$, приняв $g_{I}=1$. Изменяя только ядерный множитель, можно найти параметр $b_{M}$. Для этого необходимо выполнить расчет констант СТС в рамках двух моделей ядра: точечного магнитного диполя $\left(d_{\mathrm{nuc}}=0\right)$ и равномерно намагниченного шара $\left(d_{\text {nuc }}=1\right)$ при фиксированном радиусе ядра, тогда

$$
b_{M}=\left(R / \lambda_{C}\right)^{1-2 \gamma}\left(1-\frac{A\left(g_{I}, 1, R\right)}{A\left(g_{I}, 0, R\right)}\right) .
$$

Для того, чтобы найти параметр $b_{N}$, нужно выполнить вычисления для различных радиусов ядра $R$ :

$$
b_{N}=\frac{\left(A\left(g_{I}, 0, R_{2}\right)-A\left(g_{I}, 0, R_{1}\right)\right) \lambda_{C}^{2 \gamma-1}}{A\left(g_{I}, 0, R_{2}\right) R_{1}^{2 \gamma-1}-A\left(g_{I}, 0, R_{1}\right) R_{2}^{2 \gamma-1}} .
$$

После этого может быть найден третий параметр:

$$
\mathcal{A}_{0}=\frac{A\left(g_{I}, 0, R\right)}{g_{I}\left(1-b_{N}\left(R / \lambda_{C}\right)^{2 \gamma-1}\right)} .
$$

\section{3. Сверхтонкая магнитная аномалия}

Сравнивая константы сверхтонкой структуры для двух изотопов с ядерными $g$-факторами $g_{I}^{(1)}$ и $g_{I}^{(2)}$ и близкими ядерными радиусами $R^{(1,2)}=R \pm \mathfrak{r}$, можно вычислить значение $\mathrm{CMA}^{1} \Delta^{2}$ согласно (1). В СМА ${ }^{1} \Delta^{2}$ могут быть выделены вклады, связанные с изменением распределения заряда ${ }^{1} \Delta_{\mathrm{BR}}^{2}$ и намагниченности ${ }^{1} \Delta_{\mathrm{BW}}^{2}$ по ядру: ${ }^{1} \Delta^{2}={ }^{1} \Delta_{\mathrm{BR}}^{2}+{ }^{1} \Delta_{\mathrm{BW}}^{2}$.

Полагая ядерные факторы обоих изотопов $d_{\text {nuc }}^{(1)}=d_{\text {nuc }}^{(2)}=0$, получим

$$
\frac{A\left(g_{I}^{(1)}, 0, R+\mathfrak{r}\right)}{A\left(g_{I}^{(2)}, 0, R-\mathfrak{r}\right)} \approx \frac{g_{I}^{(1)}}{g_{I}^{(2)}}+2 \mathfrak{r} \frac{\partial A\left(g_{I}^{(1)}, 0, R\right) / \partial R}{A\left(g_{I}^{(2)}, 0, R\right)} .
$$

Тогда часть СМА, связанная с изменением распределения заряда по ядру ${ }^{1} \Delta_{\mathrm{BR}}^{2}(R)$, равна

$$
\begin{aligned}
{ }^{1} \Delta_{\mathrm{BR}}^{2}(R, \mathfrak{r}) & \equiv \frac{g_{I}^{(2)} A\left(g_{I}^{(1)}, 0, R+\mathfrak{r}\right)}{g_{I}^{(1)} A\left(g_{I}^{(2)}, 0, R-\mathfrak{r}\right)}-1 \approx \\
& \approx-2(2 \gamma-1) b_{N} \frac{R^{2 \gamma-2} \mathfrak{r}}{\lambda_{C}^{2 \gamma-1}} .
\end{aligned}
$$

Если ядерные факторы двух изотопов равны $d_{\text {nuc }}^{(1)}=d_{\text {nuc }}^{(2)}=d_{\text {nuc }} \quad$ (изотопы $\quad$ с одинаковыми спинами и похожими ядерными конфигурациями), то и для СМА ${ }^{1} \Delta^{2}$ может быть получено аналогичное выражение:

$$
\begin{aligned}
{ }^{1} \Delta^{2} & \equiv \frac{A\left(1, d_{\text {nuc }}, R+\mathfrak{r}\right)}{A\left(1, d_{\text {nuc }}, R-\mathfrak{r}\right)}-1 \approx \\
& \approx-2(2 \gamma-1)\left(b_{N}+d_{\text {nuc }} b_{M}\right) \frac{R^{2 \gamma-2} \mathfrak{r}}{\lambda_{C}^{2 \gamma-1}}
\end{aligned}
$$


Однако ядерные факторы изотопов могут достаточно сильно отличаться друг от друга $d_{\text {nuc }}^{(1)} \neq d_{\text {nuc }}^{(2)}$, тогда различием между радиусами изотопов можно пренебречь и величина СМА определяется формулой

$$
{ }^{1} \Delta^{2} \approx\left(d_{\text {nuc }}^{(2)}-d_{\text {nuc }}^{(1)}\right) b_{M}\left(\frac{R}{\lambda_{C}}\right)^{2 \gamma-1}=\epsilon_{2}-\epsilon_{1} .
$$

\section{1. Ядерный множитель}

В этом разделе обсуждаются свойства ядерного множителя $d_{\text {nuc }}$ в рамках одночастичной модели ядра. В работах $[3,16]$ было показано, что поправка БораВайскопфа может быть записана в виде:

$$
\epsilon=b_{M}\left(R / \lambda_{C}\right)^{2 \gamma-1}\left(\left(1+\frac{2}{5} \xi\right) \alpha_{S}+\frac{3}{5} \alpha_{L}\right) \frac{R_{M}^{2}}{R^{2}},
$$

где $\xi$ - параметр спиновой асимметрии:

$$
\xi= \begin{cases}\frac{2 I-1}{4(I+1)}, & \text { если } I=l+\frac{1}{2} \\ \frac{2 I+3}{4 I}, & \text { если } I=l-\frac{1}{2} .\end{cases}
$$

Коэффициенты $\alpha_{S}$ и $\alpha_{L}$ параметризуют спиновый и орбитальный вклады в ядерный $g$-фактор

$g_{I}=\alpha_{S} g_{S}+\alpha_{L} g_{L}$, тогда

$$
\alpha_{S}=\frac{g_{S}}{g_{I}} \frac{g_{I}-g_{L}}{g_{S}-g_{L}}, \quad \quad \alpha_{L}=1-\alpha_{S} .
$$

$R_{M}$ - радиус распределения плотности ядерной намагниченности.

Таким образом, для ядерного множителя $d_{\text {nuc }}$ получено следующее выражение:

$$
d_{\mathrm{nuc}}=\left(\left(1+\frac{2}{5} \xi\right) \alpha_{S}+\frac{3}{5} \alpha_{L}\right) \frac{R_{M}^{2}}{R^{2}}
$$

Ядерный фактор зависит от конфигурации нуклонов и может значительно изменяться при переходе от одного изотопа к другому. В то же время $d_{\text {nuc }}$ слабо зависит от заряда ядра $Z$.

Простейший случай $d_{\text {nuс }} \approx 1$ реализуется, когда ядерная конфигурация представлена одним валентным протоном в состоянии $s_{1 / 2}$, тогда $I=1 / 2, \xi=0, \alpha_{s}=1$ и $R_{M} \approx R$.

Для атомов щелочных металлов вклады электронных корреляций в константы СТС могут быть учтены с высокой точностью [17-20], тогда неопределенность значения ядерного множителя становится основной причиной погрешности при расчете констант СТС. Теперь приведем несколько оценок ядерного множителя для изотопа франция ${ }^{211} \mathrm{Fr}$, ядерная конфигурация которого представлена одним валентным протоном в состоянии $h_{9 / 2}[1,2]$.

1 . Используя $\quad(15)-(17), \quad$ определим $\quad \xi=\frac{2}{3}$, $\alpha_{S}=-0.152, \quad \alpha_{L}=1.152$, тогда ядерный множитель $d_{\text {nuc }}=0.50$, полагая $R_{M} \approx R, g_{L}=1$ и $g_{S}=g_{\text {free }}=5.586$.
Для изотопа ${ }^{209} \mathrm{Bi}$ с такой же ядерной конфигурацией ядерный множитель равен 0.47 [10].

2. В работе [21], следуя предположению Grossman et al. [2], использовались $g_{L}=1.16$ и $g_{S}=0.85 g_{\text {free }}$, тогда $d_{\text {nuc }}=0.33$.

3. Ядерный множитель может быть найден из отношения поправок Бора-Вайскопфа, полученных как в рамках одночастичной модели ядра, так и модели равномерно намагниченного шара. В последней модели ядерный множитель равен 1.0. Ядерный множитель, полученный таким способом, равен 0.54(21) [20].

4. Ядерный множитель $d_{\text {nuc }}=0.85$ может быть получен из отношения радиуса распределения ядерной намагниченности к радиусу ядра $\left(R_{M}=6.71 \mathrm{fm}[17]\right.$ и $R=7.281(65) \mathrm{fm}[22])$, как $d_{\mathrm{nuc}}=\left(R_{M} / R\right)^{2}$.

Представленные примеры демонстрируют, что значения ядерного множителя и поправки Бора-Вайскопфа сильно зависят от используемой ядерной модели $[23,24]$.

\section{2. Дифференциальная сверхтонкая магнитная аномалия}

В некоторых изотопических рядах отношения констант СТС для низколежащих атомных состояний $\rho=A\left(s_{1 / 2}\right) / A\left(p_{1 / 2}\right)$ измерены с точностью, достаточной чтобы надежно извлечь дифференциальную СМА:

$$
{ }_{s_{1 / 2}}^{1} \Delta_{p_{1 / 2}}^{2}=\frac{\rho^{(1)}}{\rho^{(2)}}-1 \approx{ }^{1} \Delta^{2}\left(s_{1 / 2}\right)-{ }^{1} \Delta^{2}\left(p_{1 / 2}\right) .
$$

Дифференциальные СМА между изотопами с одинаковыми ядерными спинами очень малы $\left(\sim 10^{-4}\right.$ для нейтрального атома Fr [6]). Пренебрегая этими небольшими изменениями, будем следить за перераспределением ядерной намагниченности (изменениями ядерных факторов) в случае изотопов с различными ядерными спинами. Тогда СМА описывается уравнением (13), и отношение сверхтонких магнитных аномалий

$$
\frac{{ }^{1} \Delta^{2}\left(s_{1 / 2}\right)}{{ }^{1} \Delta^{2}\left(p_{1 / 2}\right)} \approx \frac{b_{M}\left(s_{1 / 2}\right)}{b_{M}\left(p_{1 / 2}\right)} \equiv \eta
$$

определяется только атомными параметрами - атомный множитель [25,26].

Используя вычисленное значение $\eta$ и считая известным ядерный $g$-фактор $g_{I}^{(1)}$, можно восстановить СМА для состояния $s_{1 / 2}$ и вычислить поправку на СМА к $g$ фактору второго изотопа:

$$
{ }^{1} \Delta^{2}\left(s_{1 / 2}\right) \approx \frac{s_{1 / 2}^{1} \Delta_{p_{1 / 2}}^{2}}{1-1 / \eta}, \quad g_{I}^{(2)}=g_{I}^{(1)} \frac{A^{(2)}}{A^{(1)}}\left(1-{ }^{1} \Delta^{2}\left(s_{1 / 2}\right)\right) .
$$

Также может быть найден ядерный множитель $d_{\text {nuc }}^{(2)}$, если известен $d_{\text {nuc }}^{(1)}$ и измерена дифференциальная СМА:

$$
d_{\text {nuc }}^{(2)} \approx d_{\text {nuc }}^{(1)}+\frac{s_{1 / 2} \Delta_{p_{1 / 2}}^{2}}{(1-1 / \eta) b_{M}\left(s_{1 / 2}\right)\left(R / \lambda_{C}\right)^{2 \gamma-1}} .
$$


Таблица 1. Зависимость поправок Бора-Вайскопфа $\epsilon\left(d_{\text {nuc }}, R\right) \quad(5), \quad(7)$ для состояний $2 s_{1 / 2}$ и $2 p_{1 / 2}$ водородоподобных ионов и их отношения $\eta=\frac{\epsilon\left(s_{1 / 2}\right)}{\epsilon\left(p_{1 / 2}\right)}$ от заряда ядра $Z$, полагая $d_{\text {nuc }}=1$. Радиусы ядер $R$ взяты из [29]

\begin{tabular}{c|c|c|c|c|c|c|c|c}
\hline \multirow{2}{*}{$Z$} & \multicolumn{2}{|c|}{$\epsilon(1, R)\left(2 s_{1 / 2}\right), \%$} & \multicolumn{2}{c|}{$\epsilon(1, R)\left(2 p_{1 / 2}\right), \%$} & \multicolumn{2}{c}{$\eta$} \\
\cline { 2 - 8 } & $\begin{array}{c}\text { настоя- } \\
\text { щая } \\
\text { работа }\end{array}$ & {$[10]$} & {$[3]$} & $\begin{array}{c}\text { настоя- } \\
\text { щая } \\
\text { работа }\end{array}$ & {$[10]$} & {$[3]$} & $\begin{array}{c}\text { настоя- } \\
\text { щая } \\
\text { работа }\end{array}$ & {$[10]$} \\
\hline 10 & 0.042 & 0.043 & 0.05 & - & 0.0002 & - & - & 277.3 \\
20 & 0.106 & 0.106 & 0.12 & - & 0.0015 & - & - & 73.9 \\
30 & 0.203 & 0.205 & 0.23 & 0.006 & 0.006 & 0.01 & 33.1 & 33.2 \\
40 & 0.341 & 0.344 & 0.41 & 0.019 & 0.019 & 0.03 & 18.4 & 18.5 \\
50 & 0.553 & 0.561 & 0.67 & 0.048 & 0.048 & 0.08 & 11.5 & 11.6 \\
60 & 0.856 & 0.873 & 1.03 & 0.111 & 0.112 & 0.17 & 7.74 & 7.79 \\
70 & 1.335 & 1.353 & 1.51 & 0.245 & 0.245 & 0.36 & 5.45 & 5.52 \\
80 & 1.976 & 2.048 & 2.15 & 0.495 & 0.505 & 0.70 & 3.99 & 4.05 \\
90 & 2.969 & 3.077 & 2.88 & 0.987 & 1.004 & 1.27 & 3.01 & 3.07
\end{tabular}

\section{4. Результаты и обсуждение}

Этот раздел посвящен изучению общих свойств СМА. Зависимость поправки Бора-Вайскопфа от радиуса распределения ядерной намагниченности $R_{M}$ и зависимость отношения поправок Бора-Вайскопфа для состояний $s_{1 / 2}$ и $p_{1 / 2}$ от заряда ядра были изучены на примере водородоподобных ионов.

Константы СТС для водородоподобных ионов получены как аналитически, так и численно, для чего применялся пакет программ [27], который использует в качестве начального приближения программу HFD [28]. В рамках одноэлектронного приближения было получено аналитическое выражение для атомного множителя $\eta$ для нейтральных атомов. Известные в литературе результаты корреляционных расчётов атомного множителя для одновалентных атомов согласуются со значениями, полученными аналитически.

\section{1. Водородоподобные ионы}

Рисунок иллюстрирует зависимость константы СТС $A / g_{I}$ для основного состояния $\mathrm{Fr}^{86+}$ от ядерного радиуса $R$ и радиуса распределения плотности ядерной намагниченности $R_{M}$. Параметры $b_{N}\left(1 s_{1 / 2}\right)=1.241(20)$ и $b_{M}\left(1 s_{1 / 2}\right)=0.208(3)$ найдены из (8) и (7). Параметр $\mathcal{A}_{0}\left(1 s_{1 / 2}\right)$, полученный по (9), находится в согласии с аналитическим значением [10]: 398.4(6) и $397.7 \mathrm{THz}$ coответственно. Представленные на рисунке аппроксимации результатов расчетов констант СТС при различных радиусах ядра для $d_{\text {nuc }}$, равного 0 или 1, выполнены по (6) с использованием найденных выше параметров. Если зафиксировать $R=R_{0}$ и изменять $R_{M}$ от $R_{0}$ до 0 , то согласно (17) ядерный множитель $d_{\text {nuc }}\left(R_{M}\right)$ и поправка Бора-Вайскопфа будут изменяться от их максимальных значений до 0 пропорционально $R_{M}^{2}$.

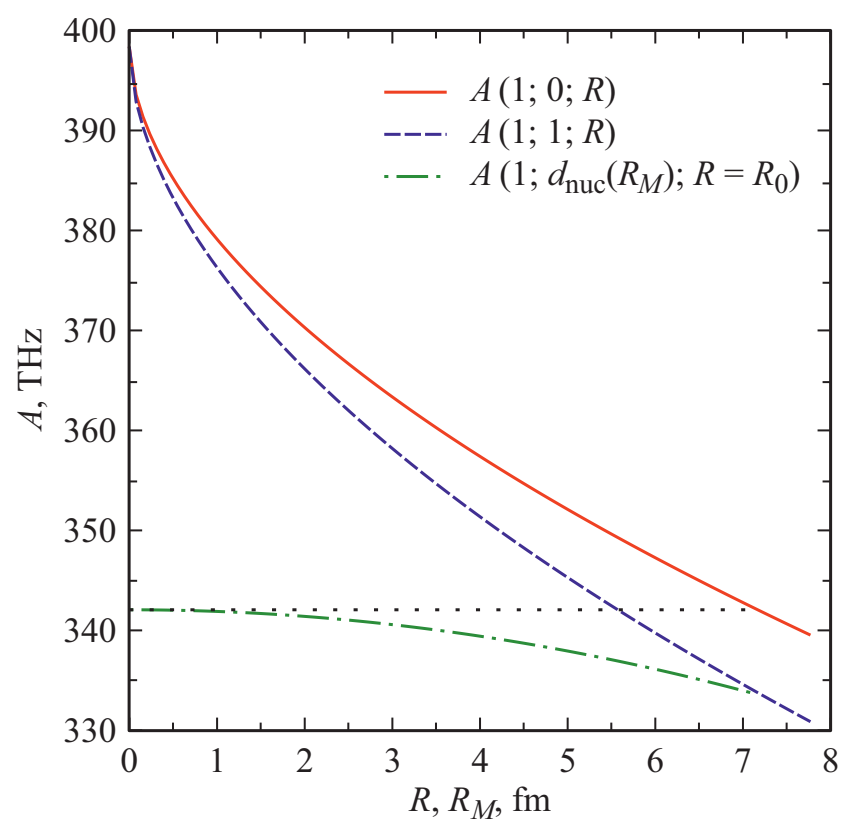

Зависимость константы СТC $A\left(g_{I}, d_{\text {nuc }}\left(R_{M}\right), R\right)$ для основного состояния водородоподобного иона $\mathrm{Fr}$ от радиуса ядра $R$ и радиуса распределения плотности ядерной намагниченности $R_{M}$ для $g_{I}=1$. Сплошная и штриховая линии описываются уравнением (6), когда $d_{\text {nuc }}=0$ и 1 соответственно. Штрихпунктирная линия сответствует случаю, когда радиус ядра $R=R_{0}$ зафиксирован, а $R_{M} \in\left[0, R_{0}\right]\left(d_{\text {nuc }} \in[0,1]\right)$. Как следует из (17), этот график - парабола с вершиной в точке $R_{M}=0$.

Таблица 2. Зависимость поправок Брейта-Розенталь $\delta(R)(5),(8)$ для состояний $2 s_{1 / 2}$ и $2 p_{1 / 2}$ водородоподобных ионов, их отношения $\eta_{B R}=\frac{\delta\left(s_{1 / 2}\right)}{\delta\left(p_{1 / 2}\right)} \quad$ и коэффициента $k=1-\delta(R)\left(2 s_{1 / 2}\right)+\delta(R)\left(2 p_{1 / 2}\right)$ от заряда ядра Z. Радиусы ядер $R$ взяты из [29]

\begin{tabular}{c|c|c|c|c|c|c|c}
\hline$Z$ & \multicolumn{2}{|c|}{$\delta(R)\left(2 s_{1 / 2}\right), \%$} & \multicolumn{2}{|c|}{$\delta(R)\left(2 p_{1 / 2}\right), \%$} & \multicolumn{2}{|c}{$\eta_{B R}$} \\
\cline { 2 - 8 } & $\begin{array}{c}\text { настоящая } \\
\text { работа }\end{array}$ & {$[10]$} & $\begin{array}{c}\text { настоящая } \\
\text { работа }\end{array}$ & {$[10]$} & $\begin{array}{c}\text { настоящая } \\
\text { работа }\end{array}$ & {$[10]$} & \\
\hline 10 & 0.115 & 0.115 & - & 0.0003 & - & 383.3 & 1.00 \\
20 & 0.299 & 0.295 & 0.0036 & 0.0036 & 82.6 & 81.9 & 1.00 \\
30 & 0.601 & 0.593 & 0.0167 & 0.0165 & 36.0 & 35.9 & 0.99 \\
40 & 1.08 & 1.06 & 0.0545 & 0.0539 & 19.8 & 19.7 & 0.99 \\
50 & 1.90 & 1.86 & 0.156 & 0.154 & 12.2 & 12.1 & 0.98 \\
60 & 3.28 & 3.20 & 0.406 & 0.400 & 8.1 & 8.0 & 0.97 \\
70 & 5.83 & 5.60 & 1.05 & 1.01 & 5.6 & 5.5 & 0.95 \\
80 & 10.1 & 9.87 & 2.56 & 2.52 & 3.9 & 3.9 & 0.92 \\
90 & 18.3 & 17.7 & 6.50 & 6.30 & 2.8 & 2.8 & 0.87
\end{tabular}

В табл. 1 и 2 приведены результаты расчетов поправок Бора-Вайскопфа (считая $d_{\text {nuc }}=1$ ) и Брейта-Розенталь состояний $2 s_{1 / 2}$ и $2 p_{1 / 2}$ водородоподобных ионов для $Z$ от 10 до 90. Вычисленные значения поправок $\epsilon$ и $\delta$ и их отношения $\eta=\frac{\epsilon\left(s_{1 / 2}\right)}{\epsilon\left(p_{1 / 2}\right)}$ и $\eta_{\mathrm{BR}}=\frac{\delta\left(s_{1 / 2}\right)}{\delta\left(p_{1 / 2}\right)}$ находятся в согласии с результатами, полученными аналитически в 
Таблица 3. Зависимость параметров $\mathcal{A}_{0}$ (9) для состояний $2 s_{1 / 2}, 2 p_{1 / 2} 2 p_{3 / 2}$ водородоподобных ионов в сравнении с аналитическими значениями, полученными по (3) [10]. В последних столбцах приведены численные значения отношения $\frac{\mathcal{A}_{0, s_{1 / 2}}}{\mathcal{A}_{0, p_{1 / 2}}}$ и $\frac{\mathcal{A}_{0, p_{1 / 2}}}{\mathcal{A}_{0, p_{3 / 2}}}$ в сравнении с аналитическими результатами, полученными по (4)

\begin{tabular}{|c|c|c|c|c|c|c|c|c|c|c|}
\hline \multirow[t]{2}{*}{$Z$} & \multicolumn{2}{|c|}{$\mathscr{A}_{0, s_{1 / 2}}, \mathrm{THz}$} & \multicolumn{2}{|c|}{$\mathscr{A}_{0, p_{1 / 2}}, \mathrm{THz}$} & \multicolumn{2}{|c|}{$\mathscr{A}_{0, p_{3 / 2}}, \mathrm{THz}$} & \multicolumn{2}{|c|}{$\frac{\mathcal{A}_{0, s_{1 / 2}}}{\mathcal{A}_{0, p_{1 / 2}}}$} & \multicolumn{2}{|c|}{$\frac{\mathcal{A}_{0, p_{1 / 2}}}{\mathcal{A}_{0, p_{3 / 2}}}$} \\
\hline & (9) & (3) & (9) & (3) & (9) & (3) & $\begin{array}{c}\text { численный } \\
\text { расчет }\end{array}$ & (4) & $\begin{array}{c}\text { численный } \\
\text { расчет }\end{array}$ & (4) \\
\hline 10 & 0.032 & 0.032 & 0.0107 & 0.0107 & 0.002 & 0.002 & 3.00 & 3.00 & 5.04 & 5.04 \\
\hline 20 & 0.266 & 0.266 & 0.088 & 0.088 & 0.017 & 0.017 & 3.01 & 3.01 & 5.18 & 5.18 \\
\hline 30 & 0.954 & 0.954 & 0.315 & 0.315 & 0.058 & 0.058 & 3.02 & 3.02 & 5.43 & 5.43 \\
\hline 40 & 2.464 & 2.463 & 0.809 & 0.809 & 0.139 & 0.139 & 3.05 & 3.04 & 5.81 & 5.81 \\
\hline 50 & 5.409 & 5.408 & 1.761 & 1.760 & 0.275 & 0.276 & 3.07 & 3.07 & 6.39 & 6.38 \\
\hline 60 & 10.909 & 10.905 & 3.509 & 3.509 & 0.484 & 0.485 & 3.11 & 3.11 & 7.23 & 7.22 \\
\hline 70 & 21.200 & 21.185 & 6.718 & 6.717 & 0.785 & 0.787 & 3.16 & 3.15 & 8.54 & 8.49 \\
\hline 80 & 41.313 & 41.261 & 12.844 & 12.841 & 1.202 & 1.205 & 3.22 & 3.21 & 10.66 & 10.57 \\
\hline 90 & 84.547 & 84.369 & 25.660 & 25.637 & 1.761 & 1.767 & 3.29 & 3.28 & 14.51 & 14.31 \\
\hline
\end{tabular}

работе [10]. Параметры $\eta$ и $\eta_{\mathrm{BR}}$ быстро уменьшаются с ростом $Z$.

Зависимость параметров $\mathscr{A}_{0}$ для состояний $2 s_{1 / 2}$, $2 p_{1 / 2}$ и $2 p_{3 / 2}$ водородоподобных ионов от заряда ядра представлена в табл. 3. Волновые функции состояний $p_{3 / 2}$ обращаются в 0 при $r=0$, таким образом поправки Бора-Вайскопфа и Брейта-Розенталь для этих состояний равны 0. В этом случае полученные из расчетов константы СТС для $g_{I}=1$ непосредственно равны $\mathscr{A}_{0}$. Для состояний $2 s_{1 / 2}$ и $2 p_{1 / 2}$ параметры $\mathscr{A}_{0}$ получены из (9). При этом параметры $\mathcal{A}_{0}$ даже в случае больших $Z$ отличаются от значений, полученных аналитически по (3), не более чем на $0.2 \%$. Отношения $\frac{\mathscr{A}_{0, s_{1 / 2}}}{\mathcal{A}_{0, p_{1 / 2}}}$ и $\frac{\mathcal{A}_{0, p_{1 / 2}}}{\mathcal{A}_{0, p_{3 / 2}}}$ с высокой точностью описываются уравнением (4).

\section{2. Тяжелые нейтральные атомы}

Для расчета констант СТС необходимо знать волновую функцию внешнего электрона на малых расстояниях, поскольку электрон-нуклонное взаимодействие быстро убывает с увеличением расстояния между электроном и ядром [30,31]. Кулоновское поле ядра на таких расстояниях можно считать неэкранированным, a радиальные волновые функции состояний $s_{1 / 2}$ и $p_{1 / 2}$ пропорциональными друг другу с коэффициентом $\frac{Z \alpha}{2}\left(1+\frac{Z^{2} \alpha^{2}}{4}\right)$ [32]. Используя пропорциональность радиальных волновых функций и оценку для $\frac{\mathscr{A}_{0, s}}{\mathscr{A}_{0, p_{1 / 2}}}=3[11]$, получим выражения для $\eta$ и $\eta_{\mathrm{BR}}$.

Согласно (6) параметр $\eta$ может быть найден как

$$
\frac{1}{\eta}=\frac{A_{s_{1 / 2}}(1,0, R)}{A_{p_{1 / 2}}(1,0, R)} \frac{A_{p_{1 / 2}}(1,0, R)-A_{p_{1 / 2}}(1,1, R)}{A_{s_{1 / 2}}(1,0, R)-A_{s_{1 / 2}}(1,1, R)} .
$$

Пренебрегая малыми поправками Брейта-Розенталь, первая дробь в выражении (22) может быть заменена на $\frac{\mathscr{A}_{0, s_{1 / 2}}}{\mathcal{A}_{0, p_{1 / 2}}}$, вторая дробь зависит от радиальных интегралов внутри ядра, где радиальные волновые функции состояний $s_{1 / 2}$ и $p_{1 / 2}$ пропорциональны друг другу, коэффициент пропорциональности для радиальных интегралов равен $\frac{\alpha^{2} Z^{2}}{4}\left(1+\frac{\alpha^{2} Z^{2}}{4}\right)^{2}$. Тогда выражение для $\eta$ имеет вид

$$
\frac{1}{\eta}=\frac{3}{4} \alpha^{2} Z^{2}
$$

Принимая во внимание вклад поправок БрейтаРозенталь $k=1-\delta\left(s_{1 / 2}\right)+\delta\left(p_{1 / 2}\right)$ и следующие слагаемые по $\alpha Z$ в выражении $\frac{\mathscr{A}_{0, s_{1 / 2}}}{\mathscr{A}_{0, p_{1 / 2}}}(4)$, получим

$$
\frac{1}{\eta}=\frac{\alpha^{2} Z^{2}}{4} 3 k\left(1+\frac{\alpha^{2} Z^{2}}{4}\right)^{2} .
$$

Зависимсть параметра $k$ от $Z$ для водородоподобных ионов представлена в табл. 2. Зависимостью атомного множителя $\eta$ от главного квантового числа $n$ можно пренебречь.

Аналогичное выражение может быть получено для отношения поправок Брейта-Розенталь:

$$
\frac{1}{\eta_{\mathrm{BR}}}=\frac{\mathscr{A}_{0, s_{1 / 2}}}{\mathscr{A}_{0, p_{1 / 2}}} \frac{\mathscr{A}_{0, p_{1 / 2}}-A_{p_{1 / 2}}(1,0, R)}{\mathscr{A}_{0, s_{1 / 2}}-A_{s_{1 / 2}}(1,0, R)} .
$$

В силу пропорциональности волновых функций для состояний $s_{1 / 2}$ и $p_{1 / 2}$ внутри ядра вторые дроби в $(22)$ и (25) равны, тогда $\frac{\eta_{\mathrm{BR}}}{\eta}=k$.

Атомные множители $\eta$, полученные для нейтральных атомов $\mathrm{Au}, \mathrm{Tl}$ и Fr аналитически по (23), (24) и из корреляционных расчётов, находятся в согласии друг с другом (табл. 4).

\section{Заключение}

На примере водородоподобных ионов исследованы поправки к константам сверхтонкой структуры на конечный размер ядра - зарядовая (Брейта-Розенталь) и 
Таблица 4. Атомные множители $\eta$ для атомов Au,Tl, Fr, полученные из уравнений (23) и (24) в сравнении с результатами расчетов водородоподобных ионов и нейтральных атомов. В случае водородоподобных ионов $\eta=\frac{\epsilon\left(2 s_{1 / 2}\right)}{\epsilon\left(2 p_{1 / 2}\right)}$, для нейтральных атомов зависимостью $\eta$ от главного квантового числа можно пренебречь

\begin{tabular}{c|c|c|c}
\hline$\eta$ & $\mathrm{Au}$ & $\mathrm{Tl}$ & $\mathrm{Fr}$ \\
\hline$(23)$ & 4.01 & 3.82 & 3.31 \\
$(24)$ & 3.69 & 3.51 & 3.07 \\
Водородоподобный ион & 4.10 & 3.86 & 3.26 \\
Нейтральный атом & $3.3^{a}$ & $3.4(2)^{c}$ & $3.1(1)^{g}$ \\
& $4.0(3)^{b}$ & $3.1^{d}$ & $3.36(5)^{h}$ \\
Эксперимент & & $2.6^{e}$ & \\
\hline
\end{tabular}

Примечание. ${ }^{a}[33] ;{ }^{b}[34] ;{ }^{c}[35] ;{ }^{d}[14] ;{ }^{e}[15] ;{ }^{f}[36] ;{ }^{g}[37] ;{ }^{h}[38]$.

магнитная (Бора-Вайскопфа). Эти поправки нарушают пропорциональность между константами сверхтонкой структуры и ядерными $g$-факторами, что получило название сверхтонкой магнитной аномалии. Распределение намагниченности по ядру может сильно меняться при переходе от одного изотопа к другому. Таким образом, главный вклад в СМА вносят магнитные поправки.

Из измеренных значений дифференциальных СМА можно восстановить их абсолютные значения, если выполнить расчёт атомного множителя $\eta$ - отношения поправок Бора-Вайскопфа для состояний $s_{1 / 2}$ и $p_{1 / 2}$. В работе зависимость атомного множителя от заряда ядра исследована в случае водородоподобных ионов и обобщена на случай нейтральных атомов.

Если выполнен расчёт атомного множителя $\eta$ и дифференциальная аномалия измерена с достаточной точностью, то точность определения $g$-факторов короткоживущих изотопов может быть увеличена более чем на порядок. Ядерный множитель $d_{\text {nuc }}$ характеризует валентную конфигурацию нуклонов. Его значение может быть получено из анализа экспериментальных данных для сложных конфигураций нуклонов, выходящих за рамки одночастичной модели ядра, и использовано для проверки различных ядерных моделей.

\section{Финансирование работы}

Работа поддержана грантом Фонда развития теоретической физики „БАЗИС“ № 17-11-136-1.

\section{Благодарности}

Авторы благодарят А.Е. Барзаха и В.М. Шабаева за полезные обсуждения.

\section{Конфликт интересов}

Авторы заявляют, что у них нет конфликта интересов.

\section{Список литературы}

[1] Zhang J., Tandecki M., Collister R. et al. // Phys. Rev. Lett. 2015. V. 115. N 4. P. 042501.

[2] Grossman J.S., Orozco L.A., Pearson M.R. et al. // Phys. Rev. Lett. 1999. V. 83. N 5. P. 935.

[3] Bohr A., Weisskopf V. F. // Phys. Rev. 1950. V. 77. P. 94-98.

[4] Rosenthal J. E., Breit G. // Phys. Rev. 1932. V. 41. P. 459-470.

[5] Crawford M.F., Schawlow A.L. // Phys. Rev. 1949. V. 76. N 9. P. 1310.

[6] Persson J. R. // At. Data Nucl. Data Tables. 2013. V. 99. N 1. P. $62-68$.

[7] Andreyev A.N., Huyse M., Van Duppen P. et al. // Nature. 2000. V. 405. N 6785. P. 430-433.

[8] Stroke H.H., Blin-Stoyle R. J., Jaccarino V. // Phys. Rev. 1961. V. 123. N 4. P. 1326.

[9] Pyykkö P., Pajanne E., Inokuti M. // Int. J. Quantum Chem. 1973. V. 7. N 4. P. $785-806$.

[10] Shabaev V. M. // J. Phys. B. 1994. V. 27. P. 5825-5832.

[11] Сушков О.П., Фламбаум В.В., Хриплович И.Б. // Опт. и спектр. 1978. T. 44. C. 3-11.

[12] Andrae D. // Phys. Rep. 2000. V. 336. N 6. P. 413-525.

[13] Büttgenbach S. // Hyperfine Interact. 1984. V. 20. N 1. P. 1-64.

[14] Mårtesson-Pendrill A.-M. // Phys. Rev. Lett. 1995. V. 74. P. 2184.

[15] Konovalova E.A., Kozlov M.G., Demidov Yu.A., Barzakh A.E. // Rad. Applic. 2017. V. 2. N 3. P. 181-185.

[16] Bohr A. // Phys. Rev. 1951. V. 81. N 3. P. 331.

[17] Safronova M.S., Johnson W.R., Derevianko A. // Phys. Rev. A. 1999 . V. 60. N 6. P. 4476.

[18] Gomez E., Aubin S., Orozco L. A. et al. // Phys. Rev. Lett. 2008. V. 100. P. 172502.

[19] Sahoo B.K., Nandy D.K., Das B.P., Sakemi Y. // Phys. Rev. A. 2015. V. 91. N 4. P. 042507.

[20] Ginges J.S.M., Volotka A.V., Fritzsche S. // Phys. Rev. A. 2017. V. 96. N 6. P. 062502.

[21] Mårtensson-Pendrill A.-M. // Hyperfine Interact. 2000. V. 127. N 1-4. P. 41-48.

[22] Johnson W.R., Soff G. // Atomic Data and Nuclear Data Tables. 1985. V. 33. N 3. P. 405-446.

[23] Shabaev V.M., Artemyev A.N., Yerokhin V.A. et al. // Phys. Rev. Lett. 2001. V. 86. N 18. P. 3959.

[24] Karpeshin F.F., Trzhaskovskaya M.B. // Nucl. Phys. A. 2015. V. 941. P. 66-77.

[25] Shabaev V.M., Glazov D.A., Oreshkina N.S. et al. // Phys. Rev. Lett. 2006. V. 96. N 25. P. 253002.

[26] Schmidt S., Billowes J., Bissell M.L. et al. // Phys. Lett. B. 2018. V. 779. P. 324-330.

[27] Kozlov M.G., Porsev S.G., Safronova M.S., Tupitsyn I.I. // Comput. Phys. Commun. 2015. V. 195. P. 199-213.

[28] Братиев В. Ф., Дайнека Г. Б., Тупицын И. И. // Изв. Акад. Наук СССР, сер. физ. 1977. Т. 41. С. 2655.

[29] Angeli I., Marinova K.P. // At. Data Nucl. Data Tables. 2013. V. 99. N 1. P. 69-95.

[30] Fermi E., Segrè E. // Zeitschrift für Physik. 1933. V. 82. N 11-12. P. 729-749.

[31] Собельман И.И. Введение в теорию атомных спектров. М.: Наука, 1977. 319 с.

[32] Хриплович И.Б. Несохранение четности в атомных явлениях. М.: Наука, 1988. 287 с. 
[33] Song S., Wang G., Ye A., Jiang G. // J. Phys. B. 2007. V. 40. N 3. P. 475.

[34] Barzakh A. E., Andreyev A. N., Monthery M. Al. et al. // Phys. Rev. C. 2020. V. 101. P. 034308.

[35] Prosnyak S.D., Maison D.E., Skripnikov L.V. // J. Chem. Phys. 2020. V. 152. N 4. P. 044301.

[36] Chen T.-L., Fan I., Chen H.-C. et al. // Phys. Rev. A. 2012. V. 86. N 5. P. 052524.

[37] Konovalova E.A., Demidov Yu.A., Kozlov M.G., Barzakh A.E. // Atoms. 2018. V. 6. N 3. P. 39.

[38] Roberts B.M., Ginges J.S.M. Nuclear magnetic moments of francium 207-213 from precision hyperfine comparisons // arXiv:2001.01907. 2020. 